\title{
SOME RESULTS ON COMPACT CONVERGENCE SEMIGROUPS DEFINED BY FILTERS
}

\author{
PHOEBE HO, PAUL PLUMMER, and SHING SO \\ Central Missouri State University \\ Warrensburg, Missouri
}

(Received June 10, 1996 and in revised form September 30, 1996)

\begin{abstract}
In this paper the concept of convergence defined by filters is used and applied in the study of semigroups. Special emphasis is placed on compact convergence semigroups and their properties.
\end{abstract}

KEY WORDS AND PHRASES: convergence semigroups, convergence groups, maximal subgroups, compact, pseudotopological, dense.

AMS(MOS) SUBJ. CLASSIFICATION CODES: Primary: 54A20, 54H11, 22M99

\section{INTRODUCTION.}

Let $\dot{x}$ be the set of all subsets of a non-empty set $S$ which contains $\{x\}$. Then $\dot{x}$ is an ultrafilter called the principle ultrafilter. In [1], Kent's approach to convergence was to set up a mapping $q$ from $F(X)$, the set of all filters on a set $X$, to $P(X)$, the power set of $X$. Then a filter $\mathcal{F}$ on $\mathrm{X}$ is said to $q$-converge to $x$ in $\mathrm{X}$, denoted by $\mathcal{F} \rightarrow \mathrm{x}$, if $\mathrm{x} \in \mathrm{q}(\mathcal{F})$.

DEFINITION. A convergence space $(X, q)$ is a non-empty set $X$ and a mapping $q$ bctween $\mathrm{F}(\mathrm{X})$ and $\mathrm{P}(\mathrm{X})$ which satisfy the following conditions:

i) $\dot{\mathrm{x}} \rightarrow \mathrm{x}$, for all $\mathrm{x} \in \mathrm{X}$;

ii) if $\mathcal{F} \rightarrow \mathrm{x}$ and $\mathcal{F} \leq \mathcal{G}$, then $\mathcal{G} \rightarrow \mathrm{x}$;

iii) if $\mathcal{F} \rightarrow \mathrm{x}$ and $\mathcal{G} \rightarrow \mathrm{x}$, then $\mathcal{F} \cap \mathcal{G} \rightarrow \mathrm{x}$.

When these properties are satisfied $q$ is known as a convergence structure. If the convergence structure is fixed for a specific discussion, as in reference to a general convergencc space, we will refer to $(X, q)$ as "the convergence space X". A convergence space $\mathrm{X}$ is Hausdorff if each filter converges to at most one point and $\mathrm{X}$ is compact if every ultrafilter converges in $\mathrm{X}$.

$\operatorname{DEFINITION}$. Let $(X, q)$ and $(Y, p)$ be convergence spaces and $f: X \rightarrow Y$. If $\mathcal{F}$ is a filter on $S$, then $f(\mathcal{F})$ will denote the filter on $Y$ with $\{f(F) \mid F \in \mathcal{F}\}$ as its base. The function $f$ is said to be continuous at $x$ if $f(\mathcal{F})$ p-converges to $f(x)$ whenever $\mathcal{F}$ q-converges to $x$.

DEFINITION. A closure operation on a convergence space $\mathrm{S}$ is defined in the following way. If $\mathrm{A} \subseteq \mathrm{S}$ and $\mathrm{x} \in \mathrm{S}$, then $\mathrm{x} \in \bar{A}$ if there exists a filter $\mathcal{F}$ such that $\mathrm{A} \in \mathcal{F}$ and $\mathcal{F} \rightarrow \mathrm{x}$. 
$\mathrm{A}$ is defined to be closed if and only if $\bar{A}=A$.

The following five lemmas are immediate results from the definitions involved.

LEMMA 1.1. If $\mathrm{A}$ is a compact subset of $\mathrm{X}$ and $f:(\mathrm{X}, \mathrm{q}) \rightarrow(\mathrm{Y}, \mathrm{p})$ is a continuous function, then $f(\mathrm{~A})$ is compact.

LEMMA 1.2. If $X$ is a compact convergence space and $T$ a closed subset of $X$, then $T$ is compact.

LEMMA 1.3. If $\mathrm{X}$ is a compact space, and $\mathcal{D}$ is a descending family of non-empty closed subsets of $X$, then $\cap \mathcal{D} \neq \emptyset$.

LEMMA 1.4. If $\mathrm{X}$ is a Hausdorff convergence space and $\mathrm{T}$ a compact subset of $\mathrm{X}$, then $\mathrm{T}$ is closed.

LEMMA 1.5. If $A$ and $B$ are compact subsets of a convergence space $X$, then $A \times B$ is compact in the product convergence structure on $\mathrm{X} \times \mathrm{X}$.

\section{MAIN RESULTS.}

DEFINITION. A convergence semigroup is a convergence space $\mathrm{S}$ together with a continuous function $m: \mathrm{S} \times \mathrm{S} \rightarrow \mathrm{S}$ such that:

i) $\mathrm{S}$ is Hausdorff;

ii) $m$ is associative.

The following notations are useful in the discussion of convergence semigroups:

i) For $\mathrm{a}, \mathrm{b} \in \mathrm{S}, \mathrm{ab}=m((\mathrm{a}, \mathrm{b}))$.

ii) For $\mathrm{A}, \mathrm{B} \subseteq \mathrm{S}, \mathrm{AB}=m(\mathrm{~A} \times \mathrm{B})=\{\mathrm{ab} \mid \mathrm{a} \in \mathrm{A}$ and $\mathrm{b} \in \mathrm{B}\}$. In particular, $\mathrm{A}\{\mathrm{b}\}$ will be denoted Ab.

iii) $\mathcal{F} \times \mathcal{G}$ is the filter on $\mathrm{S} \times \mathrm{S}$ with $\{\mathrm{F} \times \mathrm{G} \mid \mathrm{F} \in \mathcal{F}$ and $\mathrm{G} \in \mathcal{G}\}$ as its base.

iv) $\mathcal{F} \cdot \mathcal{G}$ is the filter on $\mathrm{S}$ with $m(\mathcal{F} \times \mathcal{G})$ as its base.

v) $\mathcal{F}_{\mathrm{a}}$ is the filter with $\{\mathrm{Fa} \mid \mathrm{F} \in \mathcal{F}\}$ as its base.

LEMMA 2.1. If $\mathcal{F}$ and $\mathcal{G}$ are filters on a convergence semigroup $S$ such that $\mathcal{F} \rightarrow \mathrm{x}$ and $\mathcal{G} \rightarrow \mathrm{y}$, then $\mathcal{F} \cdot \mathcal{G} \rightarrow \mathrm{xy}$.

LEMMA 2.2. If $A$ and $B$ are compact subsets of a convergence semigroup $S$, then $A B$ is compact in $\mathrm{S}$.

DEFINITION. If $f: \mathrm{X} \rightarrow \mathrm{X}$, then $\{\mathrm{x} \in \mathrm{X} \mid f(\mathbf{x})=\mathrm{x}\}$ is called the set of fixed points of $f$.

The next lemma shows two properties of fixed points in convergence spaces.

LEMMA 2.3. Let $X$ be a Hausdroff convergence space and $F$ the set of fixed points of a continuous function $f: \mathrm{X} \rightarrow \mathrm{X}$. Then the following is true:

i) If $\mathrm{F} \in \mathcal{F}$ a filter on $\mathrm{X}$, then $f(\mathcal{F})=\mathcal{F}$;

ii) If $X$ is Hausdorff, then $F$ is closed.

PROOF. i) If $\mathrm{A} \in f(\mathcal{F})$, then there exists $\mathrm{A}^{*} \in \mathcal{F}$ such that $f\left(\mathrm{~A}^{*}\right) \subseteq \mathrm{A}$. But $\mathrm{F} \in \mathcal{F}$ implies that $F \cap A^{*} \neq \emptyset$ and $F \cap A^{*} \in \mathcal{F}$. Now $F \cap A^{*} \subseteq F$ implies that $F \cap A^{*}=f\left(F \cap A^{*}\right) \subseteq$ 
$f\left(\mathrm{~A}^{*}\right) \subseteq \mathrm{A}$ which implies that $\mathrm{A} \in \mathcal{F}$. Therefore, $f(\mathcal{F}) \subseteq \mathcal{F}$.

If $\mathrm{A} \in \mathcal{F}$, then $\mathrm{A} \cap \mathrm{F} \in \mathcal{F}$ and $f(\mathrm{~A} \cap \mathrm{F})=\mathrm{A} \cap \mathrm{F}$. This implies that $\mathrm{A} \cap \mathrm{F} \in f(\mathcal{F})$. Now $\mathrm{A} \cap \mathrm{F}$ $\subseteq \mathrm{A}$ implies that $\mathrm{A} \in f(\mathcal{F})$. Therefore, $\mathcal{F} \subseteq f(\mathcal{F})$.

ii) Let $\mathrm{y} \in \overline{\mathrm{F}}$. Then there exists a filter $\mathcal{F}$ such that $\mathrm{F} \in \mathcal{F}$ and $\mathcal{F} \rightarrow \mathrm{y}$. Since $f$ is continuous, we know $\mathrm{f}(\mathcal{F}) \rightarrow f(\mathrm{y})$, and from part i), $f(\mathcal{F})=\mathcal{F}$, so $\mathcal{F} \rightarrow f(\mathrm{y})$. But X being Hausdorff implies that $f(\mathrm{y})=\mathrm{y}$. So $\mathrm{y} \in \mathrm{F}$. Therefore, $\overline{\mathrm{F}} \subseteq \mathrm{F}$ so $\overline{\mathrm{F}}=\mathrm{F}$.

DEFINITION. An element e of a semigroup $S$ is called an idempotent if $e^{2}=e$. We use $E(S)$ to denote the set of all idempotents in $S$.

DEFINITION. A nonempty subset $T$ of a semigroup $S$ is said to be a subsemigroup if $\mathrm{T} \cdot \mathrm{T}=\mathrm{T}^{2} \subseteq \mathrm{T}$. A nonempty subset $\mathrm{G}$ of $\mathrm{S}$ is a subgroup of $\mathrm{S}$ if $\mathrm{G}$ is a group under the multiplication defined on $\mathrm{S}$.

THEOREM 2.1. If a Hausdorff convergence semigroup $S$ is compact, then $S$ contains an idempotent.

PROOF. We will show that $\mathrm{S}$ contains a minimal closed subsemigroup and that every such subsemigroup consists of a single idempotent. Let $\mathcal{S}$ denote the set of closed subsemigroups of S. Note $\mathrm{S} \in \mathcal{S}$, so $\mathcal{S} \neq \emptyset$. Partially order $\mathcal{S}$ by inclusion and let $\mathcal{C}$ be a maximal tower in $\mathcal{S}$ by use of the Hausdorff Maximality Principal. Let $\mathrm{T}=\cap \mathcal{C}$. Then, from Lemma 1.3, $\mathrm{T} \neq \emptyset$. Let $x \in T$. Since $T$ is a non-empty closed subsemigroup of $S$, Lemma 1.2 implies that $T$ is compact; hence, by Lemma 2.2, xT is compact. Therefore by Lemma 1.4, xT is a closed subsemigroup of $\mathrm{S}$ contained in $\mathrm{T}$. Now, by the maximality of $\mathcal{C}$, we see that $\mathrm{xT}=\mathrm{T}$ and, similarly, $\mathrm{Tx}=\mathrm{T}$. Thus $\mathrm{T}$ is a subgroup of $\mathrm{S}$. If $\mathrm{e}$ is the identity of $\mathrm{T}$, the maximality of $\mathcal{C}$ ensures that $\mathrm{T}=\{\mathrm{e}\}$.

Let $f$ be a mapping from from the semigroup $S$ into $S \times S$ by $f(x)=(x, x)$ for all $\mathbf{x} \in \mathrm{S}$, and let $\mathcal{F}$ be a filter on $S$ such that $\mathcal{F} \rightarrow \mathrm{x}$. If $\mathrm{F} \in \mathcal{F}$, then $f(\mathrm{~F})=\{(\mathrm{x}, \mathrm{x}) \mid \mathrm{x} \in \mathrm{F}\}$. Now $\pi_{\imath}(f(\mathrm{~F}))=\mathrm{F}$ for $\mathrm{i}=1,2$, so $f(\mathcal{F}) \rightarrow(\mathrm{x}, \mathrm{x})$. Therefore $f$ is continuous. Next, consider the composite function $f \circ m$. Since $f$ and $m$ are both continuous, we know $f \circ m$ is continuous and $f \circ m: S \rightarrow S$ by $f \circ m(x)=x^{2}$. Then, by Lemma 2.2 the set of fixed points of $f \circ m$ is closed. But the set of fixed points of $f \circ m$ is $E(S)$, the set of idempotents in the semigroup. Therefore, we have the following theorem.

THEOREM 2.2. The set $E(S)$, of all idempotents of a convergence semigroup $S$, is closed.

THEOREM 2.3. Let $\mathrm{S}$ be a convergence semigroup and $\mathrm{G}$ a subgroup of $\mathrm{S}$. Then $\overline{\mathrm{G}}$ is a subsemigroup of $\mathrm{S}$ with identity.

PROOF. Let $\mathrm{x}, \mathrm{y} \in \overline{\mathrm{G}}$. This implies that there exists $\mathcal{F}$ and $\mathcal{G}$ such that $\mathrm{G}$ is contained in $\mathcal{F}$ and $\mathcal{G}$, and $\mathcal{F} \rightarrow \mathrm{x}, \mathcal{G} \rightarrow \mathrm{y}$. Since $\mathrm{G}$ is a group, $\mathrm{G} \cdot \mathrm{G}=\mathrm{G}$ so $\mathrm{G} \in \mathcal{F} \cdot \mathcal{G}$ and $\mathcal{F} \cdot \mathcal{G} \rightarrow \mathrm{xy}$. Therefore, $x y \in \overline{\mathrm{G}}$ so $\overline{\mathrm{G}}^{2} \subseteq \overline{\mathrm{G}}$.

Now if $\mathrm{e}$ is the identity of $\mathrm{G}$ and $\mathrm{x} \in \overline{\mathrm{G}}$, then there exists $\mathcal{F}$ such that $\mathrm{G} \in \mathcal{F}$ and $\mathcal{F} \rightarrow \mathrm{x}$. But since $\dot{\mathrm{e}} \rightarrow \mathrm{e}$, we know $\dot{\mathrm{e}} \cdot \mathcal{F} \rightarrow \mathrm{ex}$. Also since $\{\mathrm{e}\} \in \dot{\mathrm{e}}$ and $\mathrm{eG}=\mathrm{G}$, we have $\mathrm{G}=\mathrm{eG} \in \dot{\mathrm{e}} \cdot \mathcal{F}$. Let $F \in \mathcal{F}$. Then $G, F \in \mathcal{F}$ which implies that $\mathrm{G} \cap \mathrm{F} \in \mathcal{F}$ and since $\mathrm{G} \cap \mathrm{F} \subseteq \mathrm{G}$ we know $\mathrm{e}(\mathrm{G} \cap \mathrm{F})=\mathrm{G} \cap \mathrm{F}$ so $\mathrm{G} \cap \mathrm{F} \in \dot{\mathrm{e}} \cdot \mathcal{F}$. But $\mathrm{G} \cap \mathrm{F} \subseteq \mathrm{F}$ implies $\mathrm{F} \in \dot{\mathrm{e}} \cdot \mathcal{F}$ which means $\mathcal{F} \leq \dot{\mathrm{e}} \cdot \mathcal{F}$. 
So $\dot{e} \cdot \mathcal{F} \rightarrow \mathrm{x}$. Now $\mathrm{S}$ being Hausdorff implies that $\mathrm{ex}=\mathrm{x}$, similarly $\mathrm{xe}=\mathrm{x}$. Therefore, $\mathrm{e}$ is the identity for $\overline{\mathrm{G}}$.

THEOREM 2.4. If $S$ is a compact convergence semigroup and $G$ a subgroup of $S$, then $\overline{\mathrm{G}}$ is also a subgroup.

PROOF. By Theorem 2.3, it suffices to show if $x \in \bar{G}$ then $x$ has an inverse which is equivalent to the existence of an $x^{-1} \in \bar{G}$ such that $x^{-1} x=x x^{-1}=e$. Let $y \in \bar{G}$. Now if $y \in G$. $\mathrm{G}$ is a group so $\mathrm{y}$ has an inverse and we are done. If $\mathrm{y} \notin \mathrm{G}$, then there exists a filter $\mathcal{F}$ such that $\mathrm{G} \in \mathcal{F}$ and $\mathcal{F} \rightarrow \mathrm{y}$. For each $\mathrm{F} \subseteq \mathrm{G}$, let $\mathrm{F}^{-1}=\left\{\mathrm{x}^{-1} \mid \mathrm{x} \in \mathrm{F}\right\}$ and $\mathcal{F}^{-1}$ the filter with $\left\{\mathrm{F}^{-1} \mid \mathrm{F} \in \mathcal{F}\right.$ and $\left.\mathrm{F} \subseteq \mathrm{G}\right\}$ as its base. Now there exists an ultrafilter $\mathcal{H}$ such that $\mathcal{F}^{-1} \leq \mathcal{H}$ and, since $\mathrm{S}$ is compact, $\mathcal{H} \rightarrow \mathrm{h}$ for some $\mathrm{h} \in \mathrm{S}$. Now since $\mathrm{G} \in \mathcal{F}$ and $\mathrm{G}^{-1}=\mathrm{G}, \mathrm{G} \in \mathcal{F}^{-1}$ so $\mathrm{G} \in \mathcal{H}$ and $\mathrm{h} \in \overline{\mathrm{G}}$. By Lemma $2.1, \mathcal{H} \cdot \mathcal{F} \rightarrow$ hy so consider $\mathrm{HF} \in \mathcal{H} \cdot \mathcal{F}$. Since $\mathcal{F}^{-1} \leq \mathcal{H}$ and $F \cap G \in \mathcal{F},(F \cap G)^{-1} \in \mathcal{H}$ which implies $H \cap(F \cap G)^{-1} \in \mathcal{H}$ and $H \cap(F \cap G)^{-1} \neq \emptyset$. Thus there exists $x^{-1} \in(F \cap G)^{-1}$ such that $x^{-1} \in H$ and $x \in(F \cap G) \subseteq F$ so $x^{-1} x=e \in H F$, so $\mathrm{e} \in \mathrm{HF}$ for all $\mathrm{HF} \in \mathcal{H} \cdot \mathcal{F}$ which implies $\mathcal{H} \cdot \mathcal{F} \leq \dot{\mathrm{e}}$. Therefore, $\dot{\mathrm{e}} \rightarrow$ hy and we know $\dot{\mathrm{e}} \rightarrow \mathrm{e}$. but the fact that $\mathrm{S}$ is Hausdorff implies that hy $=$ e. Similarly, we can show yh $=$ e. Thus, $\mathrm{h}=\mathrm{y}^{-1} \in \mathrm{G}$.

DEFINITION. Let $G$ be a subgroup of a convergence semigroup $S$. If the inversion mapping $f: G \rightarrow G$ defined by $f(x)=x^{-1}$ is continuous, then $G$ is called a convergence semigroup.

DEFINITION. A convergence space $\mathrm{X}$ is said to be pseudotopological if a filter $\mathcal{F}$ converges to some point $\mathrm{x}$ in $\mathrm{X}$ if and only if all ultrafilters finer than $\mathcal{F}$ converge to $\mathrm{x}$.

THEOREM 2.5. If $S$ is a compact pseudotopological semigroup, which is algebraically a group, then $\mathrm{S}$ is a convergence group.

PROOF. Let $\mathrm{f}: \mathrm{S} \rightarrow \mathrm{S}$ defined by $\mathrm{f}(\mathrm{x})=\mathrm{x}^{-1}$ and $\mathcal{F}$ a filter such that $\mathcal{F} \rightarrow \mathrm{x}$. Then $\mathrm{f}($ $\mathcal{F})$ is a filter in $\mathrm{S}$. Let $\mathcal{H}$ be a ultrafilter such that $\mathcal{H} \geq \mathbf{f}(\mathcal{F})$. Since $\mathrm{S}$ is compact, $\mathcal{H} \rightarrow y$ for some $y$ in S. Since for every $H \in \mathcal{H}, H \cap \mathrm{f}(\mathcal{F}) \neq \emptyset$ for all $F \in \mathcal{F}$. Then $e \in H F$ for all $H \in \mathcal{H}$ and $\mathrm{F} \in \mathcal{F}$ where $e$ is the identity of the group S. Now $\mathcal{F} \mathcal{H} \rightarrow y, \mathcal{F} \mathcal{H} \leq \dot{e}$, and $\dot{e} \rightarrow e$ imply that $x y=e$.

Similarly, $y x=e$. Thus $y=x^{-1}$. Since $\mathrm{S}$ is pseudotopological, $\mathrm{f}(\mathcal{F}) \rightarrow x^{-1}$ so $\mathrm{f}$ is continuous. Therefore, $\mathrm{S}$ is a convergence group.

DEFINITION. A subset $D$ of a convergence space $X$ is said to be dense in $X$ if $\bar{D}=X$.

DEFINITION. If $S$ is a convergence semigroup and $e \in E(S)$, then the largest proper subgroup of $\mathrm{S}$ which contains $\mathrm{e}$ is called the maximal subgroup of $\mathrm{S}$ containing $\mathrm{e}$.

THEOREM 2.6. i) If $\mathrm{S}$ is a compact convergence semigroup, then each maximal subgroup is either closed or dense in $\mathrm{S}$.

ii) If $\mathrm{S}$ is a compact pseudotopological semigroup, then either every maximal subgroup of $\mathrm{S}$ is closed or $\mathrm{S}$ is a convergence group.

iii) If $\mathrm{S}$ is a compact pseudotopological semigroup, then every maximal subgroup of $\mathrm{S}$ is a 
convergence group.

PROOF. i) By Theorem 2.4, if $G$ is a subgroup then so is $\bar{G}$. Since $G \subset \bar{G} \subset S, \bar{G}$ is a group, and $G$ is maximal, either $\bar{G}=G$ and $G$ is closed, or $\bar{G}=S$ and $G$ is dense.

ii) If $S$ does have a dense subgroup, then $\bar{G}=S$ implies $S$ is a group. Since $S$ is compact and pseudotopological, $\mathrm{S}$ is a convergence semigroup by Theorem 2.5 .

iii) Let $G$ be a maximal subgroup of $S$. Then $G$ is either closed or dense in $S$.

If $\mathrm{G}$ is closed, then $\mathrm{G}$ satisfies all the hypothesis of Theorem 2.5. so $\mathrm{G}$ is a convergence group.

If $G$ is dense in $S$, then by ii) $S$ is a convergence group. Since the inversion mapping on $G$ is a restriction of the inversion mapping on $\mathrm{S}$, it must be continuous. Therefore $\mathrm{G}$ is a convergence group.

\section{REFERENCES}

1. KENT, D.C., Convergence Functions and Related Topologies, Fundamenta Mathematicae 54 (1964), 125-133.

2. WILLARD, S., General Topology, Addison-Wesley, Reading, Massachusetts, 1970.

3. PAALMAN-DE MIRANDA, A.B., Topological Semigroups, Mathematical Centre Tracts, 2nd Ed., Mathematiche Centrum, Amsterdam, 1970.

4. CARRUTH, J.H., HILBEBRANT, J.A., and KOCH, R.J., The Theory of Topological Semigroups, Marcel Dekke, Inc., New York, 1983.

5. HUSAIN, T., Introduction to Topological Groups, W. B. Saunders Company, Philadelphia and London, 1966.

6. PLUMMER, P.J., Convergence Semigroups in Convergence Spaces Defined by Filters, Thesis, Central Missouri State University, 1995. 


\section{Mathematical Problems in Engineering}

\section{Special Issue on}

\section{Modeling Experimental Nonlinear Dynamics and Chaotic Scenarios}

\section{Call for Papers}

Thinking about nonlinearity in engineering areas, up to the 70 s, was focused on intentionally built nonlinear parts in order to improve the operational characteristics of a device or system. Keying, saturation, hysteretic phenomena, and dead zones were added to existing devices increasing their behavior diversity and precision. In this context, an intrinsic nonlinearity was treated just as a linear approximation, around equilibrium points.

Inspired on the rediscovering of the richness of nonlinear and chaotic phenomena, engineers started using analytical tools from "Qualitative Theory of Differential Equations," allowing more precise analysis and synthesis, in order to produce new vital products and services. Bifurcation theory, dynamical systems and chaos started to be part of the mandatory set of tools for design engineers.

This proposed special edition of the Mathematical Problems in Engineering aims to provide a picture of the importance of the bifurcation theory, relating it with nonlinear and chaotic dynamics for natural and engineered systems. Ideas of how this dynamics can be captured through precisely tailored real and numerical experiments and understanding by the combination of specific tools that associate dynamical system theory and geometric tools in a very clever, sophisticated, and at the same time simple and unique analytical environment are the subject of this issue, allowing new methods to design high-precision devices and equipment.

Authors should follow the Mathematical Problems in Engineering manuscript format described at http://www .hindawi.com/journals/mpe/. Prospective authors should submit an electronic copy of their complete manuscript through the journal Manuscript Tracking System at http:// mts.hindawi.com/ according to the following timetable:

\begin{tabular}{|l|l|}
\hline Manuscript Due & December 1,2008 \\
\hline First Round of Reviews & March 1, 2009 \\
\hline Publication Date & June 1,2009 \\
\hline
\end{tabular}

\section{Guest Editors}

José Roberto Castilho Piqueira, Telecommunication and Control Engineering Department, Polytechnic School, The University of São Paulo, 05508-970 São Paulo, Brazil; piqueira@lac.usp.br

Elbert E. Neher Macau, Laboratório Associado de Matemática Aplicada e Computação (LAC), Instituto Nacional de Pesquisas Espaciais (INPE), São Josè dos Campos, 12227-010 São Paulo, Brazil ; elbert@lac.inpe.br

Celso Grebogi, Center for Applied Dynamics Research, King's College, University of Aberdeen, Aberdeen AB24 3UE, UK; grebogi@abdn.ac.uk 